\title{
EchoGéo
}

45 | 2018

Déclinaisons géographiques du changement social en Iran

\section{Les nouvelles irrigations pressurisées : un défi pour la cartographie des réseaux d'irrigation}

\section{Émilie Lavie}

\section{(2) OpenEdition}

\section{Journals}

Édition électronique

URL : https://journals.openedition.org/echogeo/15911

DOI : 10.4000/echogeo.15911

ISSN : 1963-1197

Éditeur

Pôle de recherche pour l'organisation et la diffusion de l'information géographique (CNRS UMR 8586)

Référence électronique

Émilie Lavie, «Les nouvelles irrigations pressurisées : un défı pour la cartographie des réseaux d'irrigation », EchoGéo [En ligne], 45 | 2018, mis en ligne le 05 novembre 2018, consulté le 10 août 2021. URL : http://journals.openedition.org/echogeo/15911 ; DOI : https://doi.org/10.4000/echogeo. 15911

Ce document a été généré automatiquement le 10 août 2021.

EchoGéo est mis à disposition selon les termes de la licence Creative Commons Attribution - Pas d'Utilisation Commerciale - Pas de Modification 4.0 International (CC BY-NC-ND) 


\title{
Les nouvelles irrigations pressurisées : un défi pour la cartographie des réseaux d'irrigation
}

\author{
Émilie Lavie
}

\section{Introduction}

1 Les espaces irrigués ont la particularité d'avoir été, sinon construits, au moins modifiés par l'alimentation en eau. Les paysages sont souvent marqués par une plus grande densité de végétation par rapport aux espaces environnants, mais pas toujours. En effet, l'irrigation se pratique dans des milieux très divers et il ne faut pas systématiquement l'associer aux espaces qui manquent d'eau (Cantor, 1968). Ainsi, dans des domaines climatiques de mousson par exemple, la gestion de l'eau et l'irrigation en terrasses permettent de cultiver même pendant la saison des pluies ; en outre en saison sèche, elle permet une troisième récolte de riz ou d'autres cultures comme des céréales. En Méditerranée, l'irrigation permet de s'affranchir ou de limiter les effets des extrêmes hydro-climatiques et surtout la baisse des précipitations en saisons culturales (Aspe, 2012). Dans les espaces arides et semi-arides, l'irrigation marque la limite entre l'oasis et les déserts environnants (Mainguet, 2003, entre autres).

2 Comme d'autres objets géographiques, les espaces irrigués ne sont pas toujours très bien individualisés dans les paysages, notamment dans des milieux relativement bien arrosés où il est difficile de singulariser les périmètres alimentés en eau de ceux qui ne le sont pas. Ils suivent aussi des dynamiques analogues à d'autres territoires en se spécialisant, afin le plus souvent de mieux s'insérer dans la mondialisation des échanges économiques (Marshall et Lavie, 2017). Ainsi, des paysages de rizières en terrasses ou des espaces oasiens sont considérés comme des patrimoines et mis en valeur pour attirer les touristes internationaux, tandis que de grands systèmes irrigués 
favorisent des produits d'exportation comme les dattes dans les Suds tunisiens et marocains, les fruits et légumes de contre-saison au Pérou, la truffe et le palmier d'ornement en Espagne ou le vin du Nouveau monde (en Californie, Chili, Argentine, Afrique du sud, Australie, etc.) ou du Vieux continent (Italie, Espagne, France, etc.).

Afin d'observer ces dynamiques de spécialisation et les enjeux politiques associés, parmi les méthodes utilisées par les géographes, il y a la cartographie des réseaux d'irrigation. Elle a cela d'intéressant qu'elle permet de mieux interpréter les mutations paysagères, de l'échelle de la parcelle à celle du système irrigué, souvent régies par des modifications de structures de distribution. La circulation de l'eau, même si elle peut s'appuyer sur la topographie, est déterminée par les gestionnaires ou des irrigants individuels ou associés. Observer le chemin de l'eau permet de ce fait d'identifier les enjeux politiques, économiques et sociaux de sa distribution, comme par exemple distinguer des interconnexions entre systèmes techniques ou regarder les interactions entre acteurs. La cartographie, au-delà de la simple représentation graphique, offre donc au géographe une porte d'entrée vers l'analyse des jeux de pouvoirs. Dessiner les réseaux d'irrigation a souvent été facile puisque les éléments techniques s'identifient bien dans le paysage ; cela passe principalement par des relevés de terrain à l'échelle fine ou le recours à des images satellitaires à petite échelle. Or, les nouvelles techniques d'irrigation sous pression sont plus difficiles à reconnaître in situ comme à distance. C'est cette difficulté rencontrée et les alternatives proposées, que je souhaiterais mettre en évidence dans cet article.

\section{Des infrastructures d'irrigation visibles dans le paysage}

\section{Des sources d'eau allogènes}

4 Si on peut pomper in situ dans les nappes, de grands systèmes irrigués dépendent de sources allogènes, c'est-à-dire prises à distance et transportées. Les infrastructures sont donc souvent assez lourdes et visibles dans le paysage.

5 Les plus marquants sont les systèmes alliant barrages de régulation, barrage de dérivation et canaux. En effet, afin de stocker les ressources, par exemple lors de saisons fraîches ou de saisons des crues, pour pouvoir disposer d'eau lors des saisons culturales, de grands barrages régulent les cours d'eau; c'est le cas du barrage d'Assouan en Egypte par exemple. D'autres infrastructures sont de moins grande emprise et ont été construites pour dériver l'eau vers les espaces de culture, via de grands canaux. Ainsi, dans la Cordillère des Andes argentines, a été finalisé en 2004 le barrage régulateur de Potrerillos (voir illustration 1) qui stocke les eaux hivernales et de fonte des neiges printanières pour les distribuer en fonction des besoins des agriculteurs dans l'oasis de Mendoza, en aval. Cette photo, prise en novembre soit au tout début de l'été, montre un lac qui n'est pas au maximum de sa capacité de remplissage, avec des marques visibles sur les rives. La fonte des neiges andines va encore se poursuivre quelques semaines et remplir la retenue. Sur le piémont, le barrage dérivateur de Cipolletti (voir illustration 2) détourne les eaux de la rivière vers l'oasis. Cette photo est prise depuis la rive gauche du cours d'eau en regardant l'aval, le canal partant vers la gauche de l'image. De l'autre côté du barrage, on peut voir à travers les piles que la rivière est à sec, toute l'eau est dérivée. 
Illustration 1 - Le lac du barrage régulateur de Potrerillos en amont de l'oasis de Mendoza, Argentine

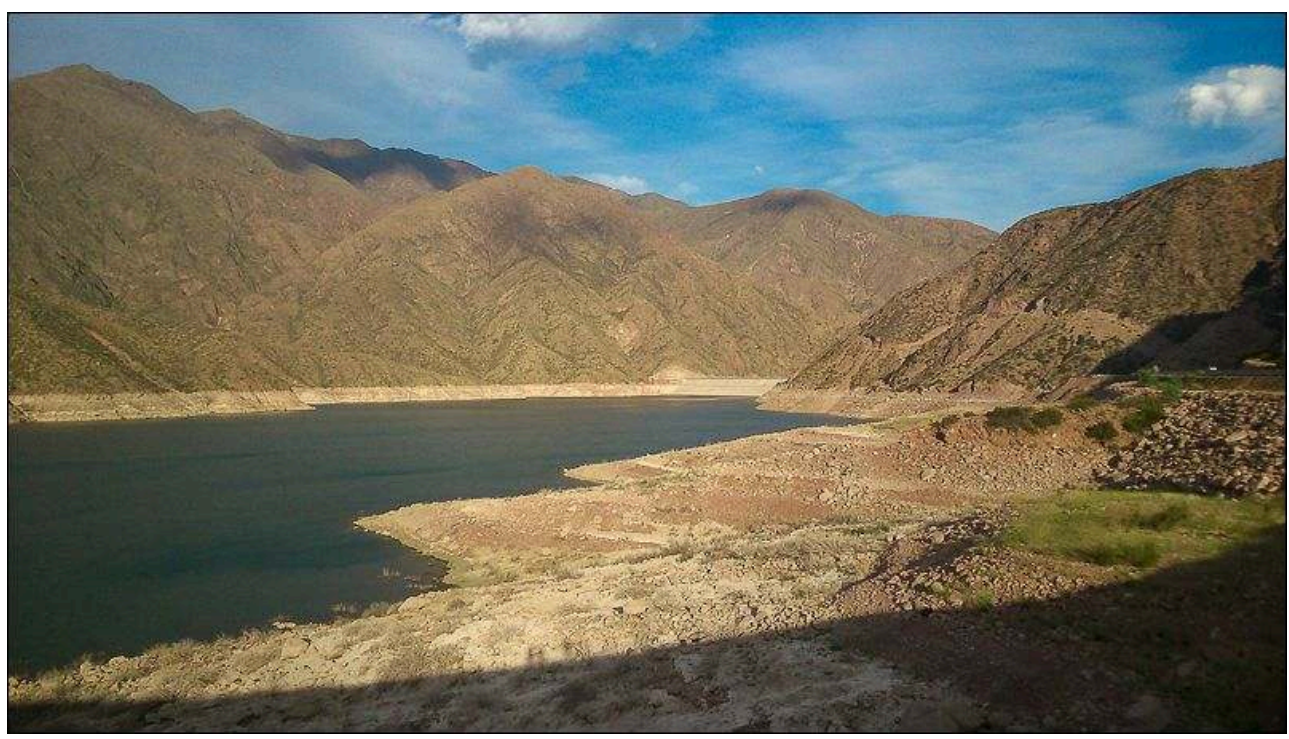

Auteur : Émilie Lavie, novembre 2014.

Illustration 2 - Le barrage dérivateur de Cipolletti à l'entrée de l'oasis de Mendoza, Argentine

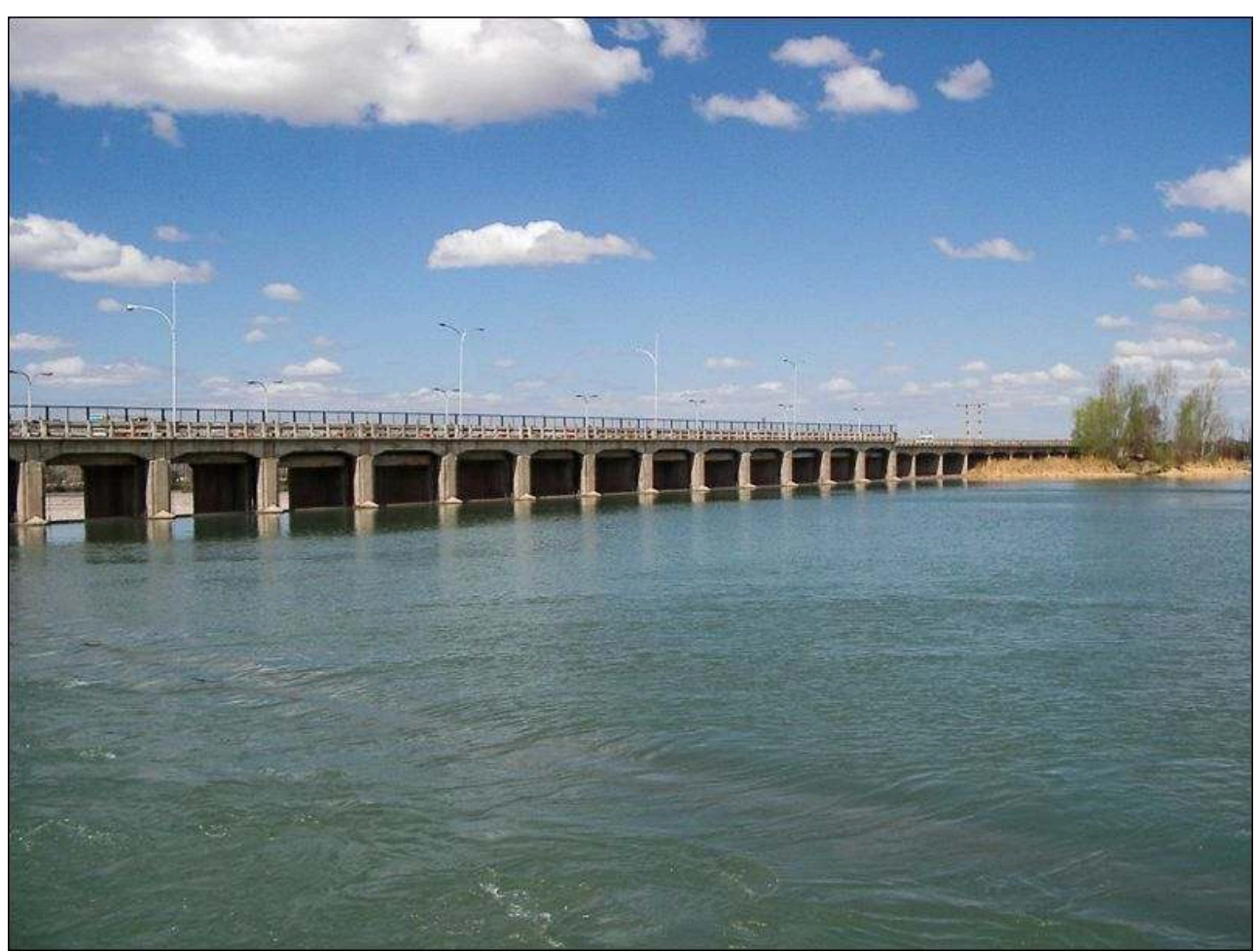

Auteur : Émilie Lavie, novembre 2007.

Dans le cas d'irrigation gravitaire, la topographie limite les espaces irrigués de ceux qui ne le sont pas puisque l'eau ne remonte pas les pentes. Par exemple à Ouaouizerth dans l'Atlas marocain, l'illustration 3 montre un canal, une seguia, au centre, qui irrigue la partie aval à droite, ici pour arroser des oliviers, tandis qu'en amont à gauche les terres 
sont à nu. Le résultat est que même sans connaitre l'emplacement exact des canaux, par exemple avant de commencer les relevés de terrain, on sait à distance ou par image satellitaire, où passent les seguias les plus hautes.

Illustration 3 - Seguia marquant la limite amont de la zone irriguée à Ouaouizerth, Maroc

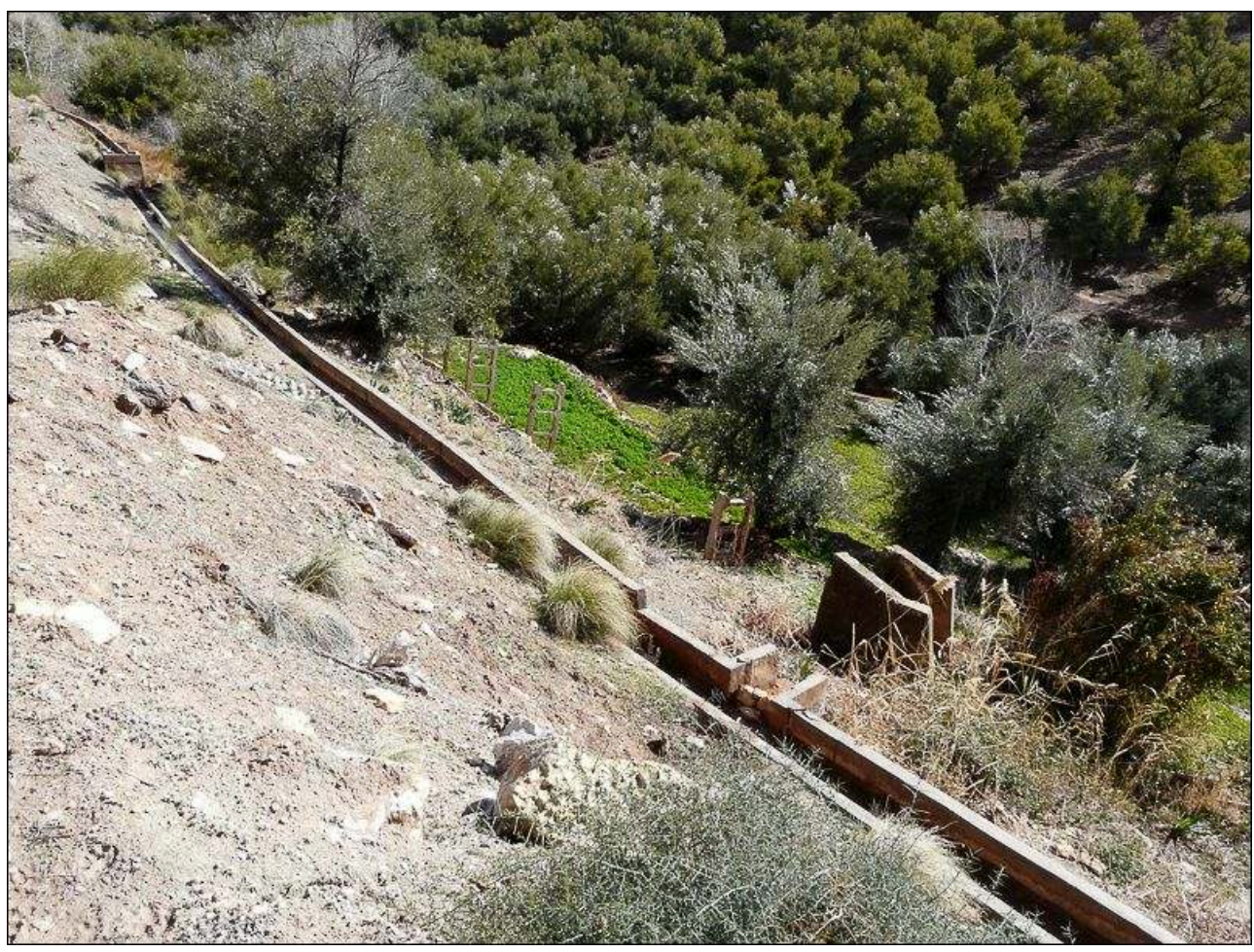

Auteur : Émilie Lavie, février 2018

Dans les exemples de Mendoza ou de Ouaouizerth, les eaux sont détournées des rivières à l'entrée de l'oasis. Dans d'autres cas, comme sur la côte péruvienne, de grands canaux dérivent la ressource en eau sur de très grandes distances. C'est le cas du canal Chavimochic au Pérou, dans le cadre du projet PECH. Ce projet a été mis en place par l'État péruvien, afin d'étendre des périmètres de culture dans le désert, via la dérivation des eaux des rivières Chao, Virú, Moche et Chicama, qui donnent leur nom au canal (Marshall, 2011). Ce canal prévu pour une longueur totale de $285 \mathrm{~km}$ une fois terminé, devrait étendre les périmètres irrigués de 93000 à 132000 ha (Marshall, 2014, p. 67).

8 Ces systèmes d'irrigation sont très visibles en surface. Mais on peut aussi capter une ressource allogène et la conduire de manière souterraine. C'est le cas des foggaras ou khettaras au Maghreb par exemple, appelées aussi qanat en Mésopotamie. Un affleurement de nappe est détourné dans de grandes galeries qui alimentent les espaces irrigués. Or ces conduites, bien que souterraines, sont très visibles depuis la surface parce que les cônes de déblais marquent le trajet de l'eau (voir illustration 4); perceptibles sur des images satellitaires, ces cônes se voient aussi très bien lorsque l'on est sur le terrain, comme sur la photo du bas. Ils ont l'avantage de permettre aux gestionnaires ou aux agriculteurs de contrôler les débits et au besoin de descendre effectuer les réparations, notamment pour désobstruer des canalisations. 
Illustration 4 - Foggaras visibles au nord d'Erfoud, Maroc

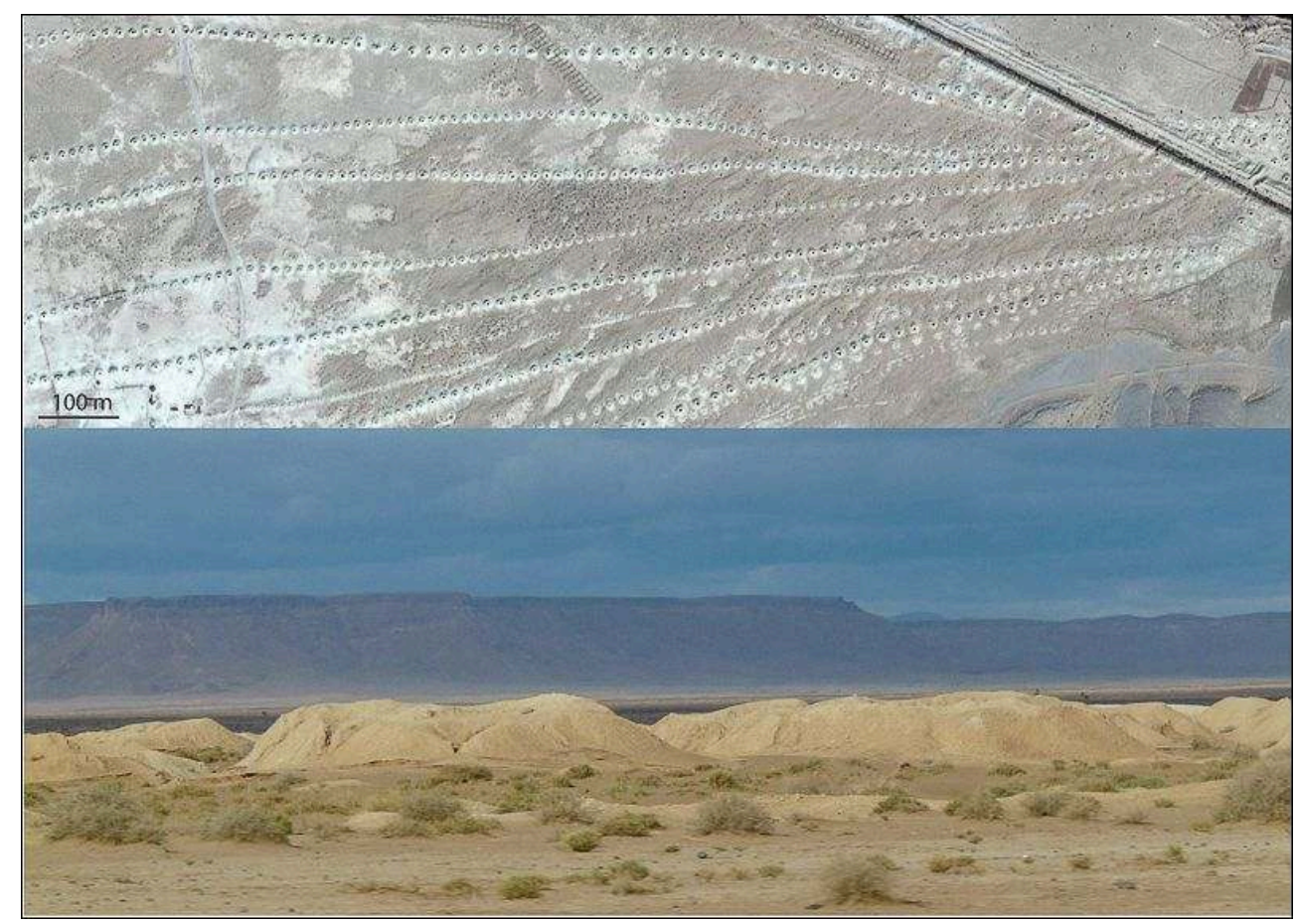

Source : image Google Earth, 2018 ; photo Émilie Lavie, janvier 2013.

\section{L'irrigation rivulaire des cours d'eau}

Depuis l'avènement des ressources en hydrocarbures, le recours au pompage directement dans les cours d'eau permet de prélever en fonction des besoins. Cette technique revêt l'avantage d'affranchir les agriculteurs de l'entretien de grandes infrastructures comme les barrages et les canaux. Cela est aussi très utile pour des terres plus hautes que les sources d'adduction. Les Égyptiens de l'Antiquité utilisaient d'ailleurs le système des chadoufs pour faire remonter l'eau.

Dans la plaine de la Gezirah au Soudan, l'eau est dérivée dans les Nils Bleu et Blanc puis distribuée à travers des canaux. Mais la platitude du terrain fait qu'il est difficile de distribuer de manière gravitaire. Afin de faire remonter l'eau dans leurs parcelles, les agriculteurs de la Gezirah ont recours aux motopompes (Illustration). Sur les rives du fleuve Sénégal, c'est pour les mêmes raisons que le pompage se fait directement dans le fleuve, soit par des irrigants privés, soit par des groupements d'agriculteurs (Bruckmann, 2016). 


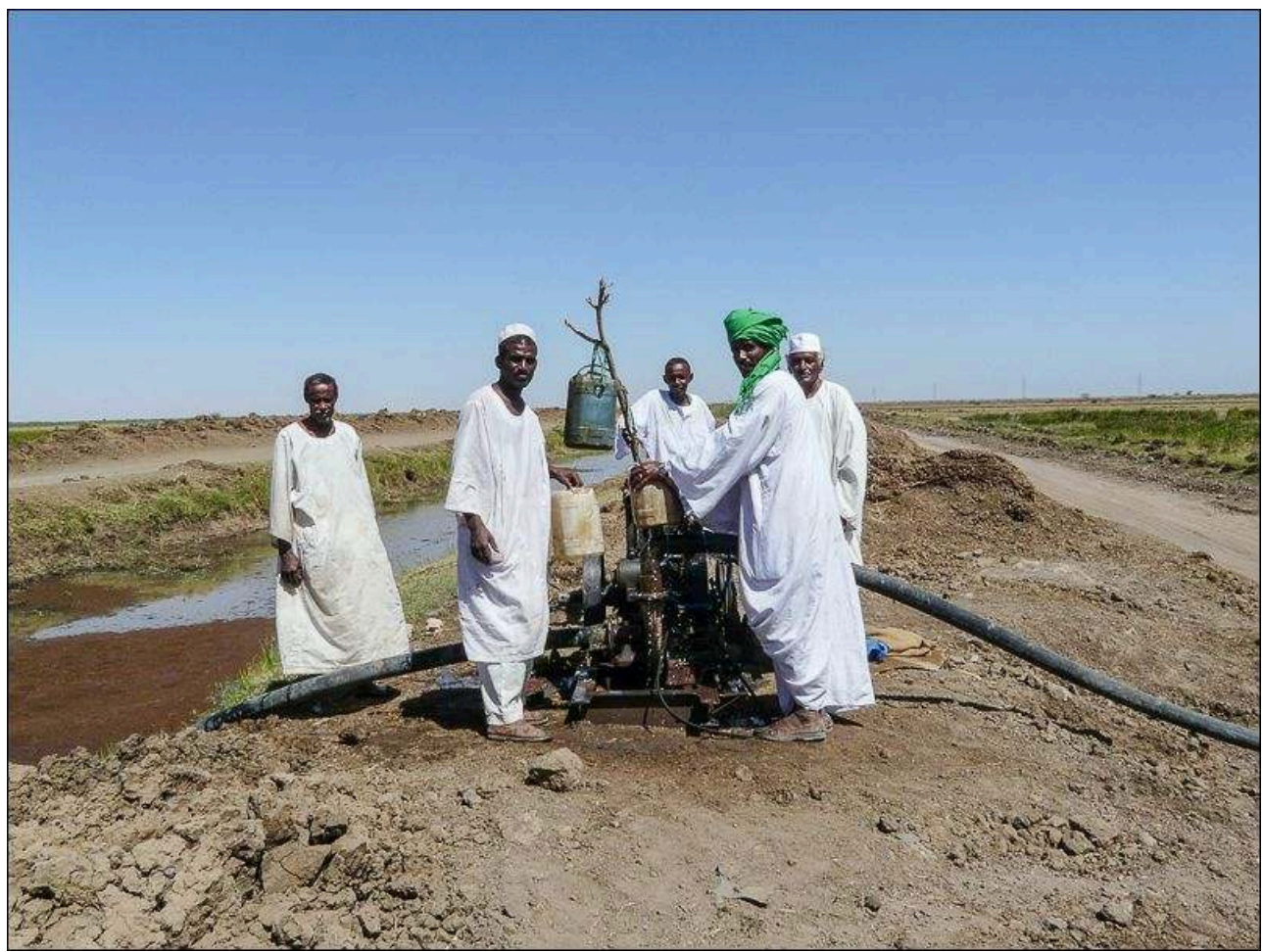

Auteur : Émilie Lavie, mars 2010.

11 Les indices des réseaux d'irrigation sont donc relativement marqués dans le paysage, d'autant plus si la ressource est allogène. Généralement elle est aussi gérée de manière collective. À l'instar des pompages directs dans les cours d'eau, des pompages dans les nappes sont moins visibles sur le terrain, notamment s'il s'agit d'alternatives personnelles et non collectives. Dans ce cas, il est pratique pour le/la chercheur.e de regarder à l'échelle des parcelles.

\section{À l'échelle des parcelles, une pluralité des systèmes}

Avant l'arrivée des motopompes et de l'irrigation pressurisée, la distribution à l'échelle des parcelles s'est souvent faite de manière gravitaire. Le détournement de l'eau en amont et son cheminement dans des canaux jusqu'à la parcelle se fait via des tours d'eau, plus rarement à la demande.

L'irrigation gravitaire en terrasses est clairement une des images d'Épinal de l'Asie des moussons. À Hélambu au Népal, la rivière est court-circuitée : l'eau est dérivée sur les rives, descend par gravité de terrasses agricoles en terrasses agricoles qui sont entièrement inondées, ici pour la culture du riz (Illustration). Les surplus retournent à la rivière, soit par des drains, soit par l'intermédiaire de la nappe alluviale pour l'eau qui s'est infiltrée. Un autre type d'irrigation gravitaire est l'ennoiement, non pas de la parcelle, mais des sillons. Les plantes, sur les billons et leurs racines peuvent pomper l'eau nécessaire. C'est le cas de cet oignon à Mendoza (Illustration) cultivé pour ses graines qui seront récoltées pour être vendues, puis plantées pour en récolter cette fois-ci, les bulbes destinés au marché local. 
Illustration 6 - Terrasses rizicoles de la vallée d'Hélambu, Népal

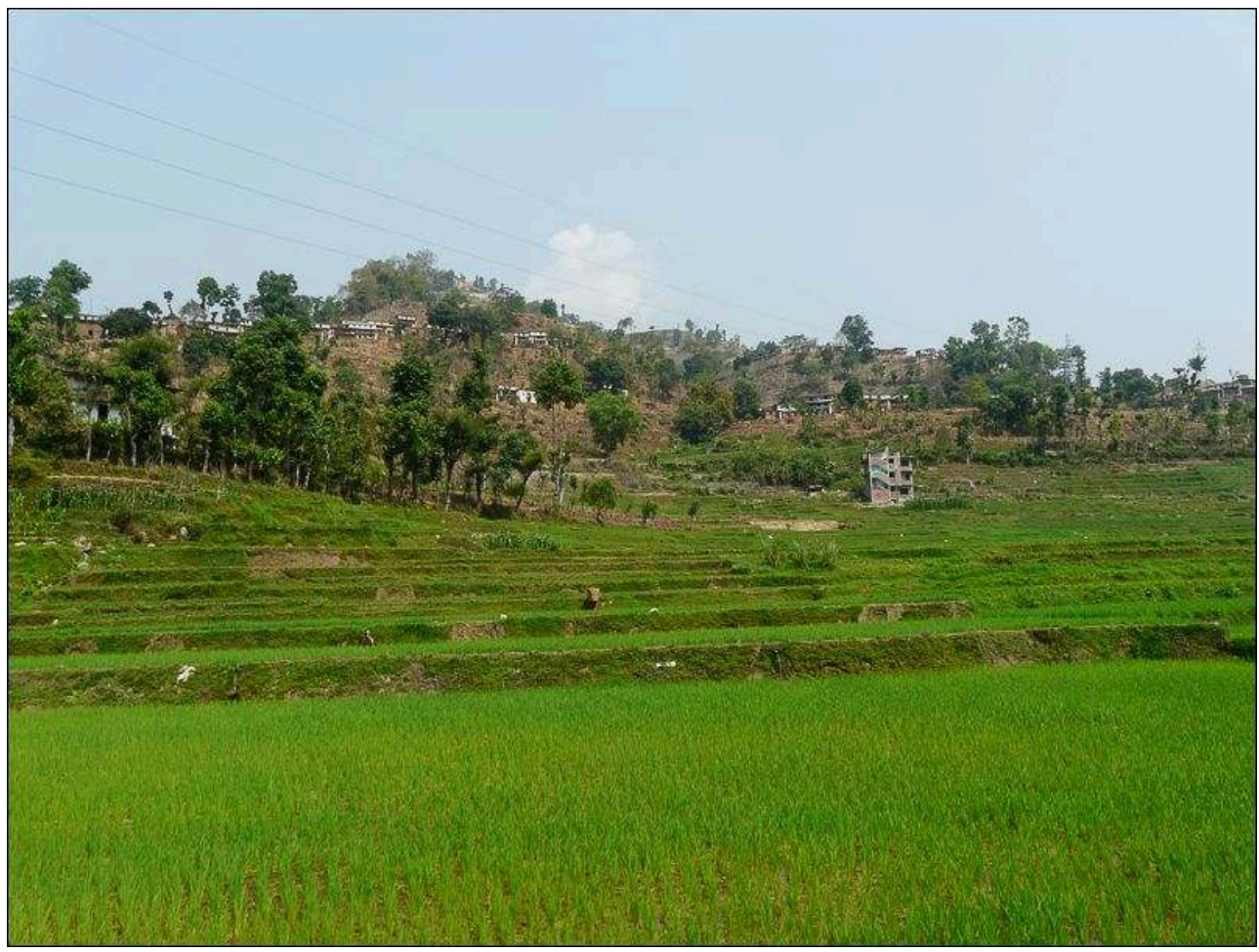

Source : Émilie Lavie, avril 2013

Illustration 7 - Irrigation gravitaire en sillons d'oignons à semences, Mendoza, Argentine

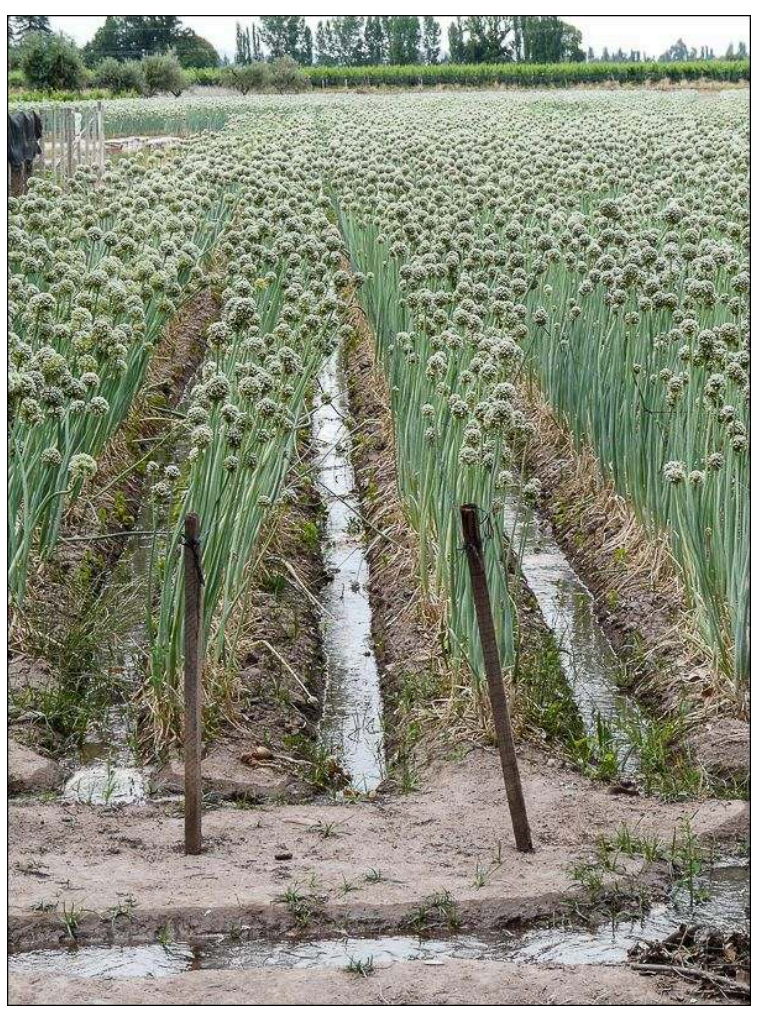

Source : Émilie Lavie, novembre 2014. 


\section{Une irrigation pressurisée qui se développe} racines, elle est sous pression. L'irrigant s'affranchit donc de fait de la gravité. Par exemple en Valle de Uco en Argentine, le tuyau de goutte-à-goutte est installé sur le pied de la vigne et les gouttes tombent sur le sol. Protégée le plus souvent du soleil par le feuillage, l'eau coule plus vite dans les sols et alimente les racines des ceps en limitant au maximum l'évaporation directe. Lorsque cela est nécessaire ou voulu, les intrants (engrais ou pesticides) sont directement apportés par le goutte-à-goutte, ce qui évite une manœuvre agricole dans les parcelles, fait baisser le coût de main d'œuvre et limite en théorie des gaspillages. Mais surtout, l'absence de l'irrigation gravitaire permet de ne pas avoir à terrasser la parcelle avant plantation, à ne pas avoir à l'aplanir et lui donner une légère pente. Les paysages gardent donc un aspect vallonné (voir illustration 8) que seules les nouvelles parcelles en irrigation pressurisée peuvent offrir. Le touriste vient ici y voir un paysage de vigne vallonné, un peu à l'européenne, qui n'existe que depuis peu en Amérique latine (Lavie et al., 2017).

Le goutte-à-goutte et la micro-aspersion se développent de plus en plus pour la culture des arbres fruitiers ou comme dans le cas de l'illustration 9, des oliviers. Cet arbre ayant peu besoin d'eau, le goutte-à-goutte est suffisant car moins cher que la microaspersion. En effet, en micro-aspersion le tuyau est agrémenté de petites douchettes; tandis que pour le goutte-à-goutte, de petites buses ou un simple trou dans le tuyau suffisent. Ici un sillon est creusé autour du pied et ennoie une zone qui permet aux racines de s'alimenter. Les tuyaux, souples, peuvent passer de parcelle en parcelle. Ils ne sont pas fixés aux plantes, c'est aussi un avantage car il en faut moins. Les sols entre les arbres sont bien secs, le gaspillage est plus limité qu'avec l'irrigation gravitaire, en particulier dans des sols perméables comme ici. Pour d'autres plantes comme les vergers ou la luzerne, la micro-aspersion est préférable. Elle est apportée au pied, comme le goutte à goutte, mais il faut que la culture soit de bonne valeur ajoutée, comme les fruits par exemple. Dans le cas de plantes fourragères (Illustration), elle arrive par la canopée, imitant la pluie. C'est aussi le principe du canon. 
Illustration 8 - Irrigation en goutte à goutte de la vigne, mettant en valeur l'aspect vallonné du paysage, Valle de Uco, Argentine

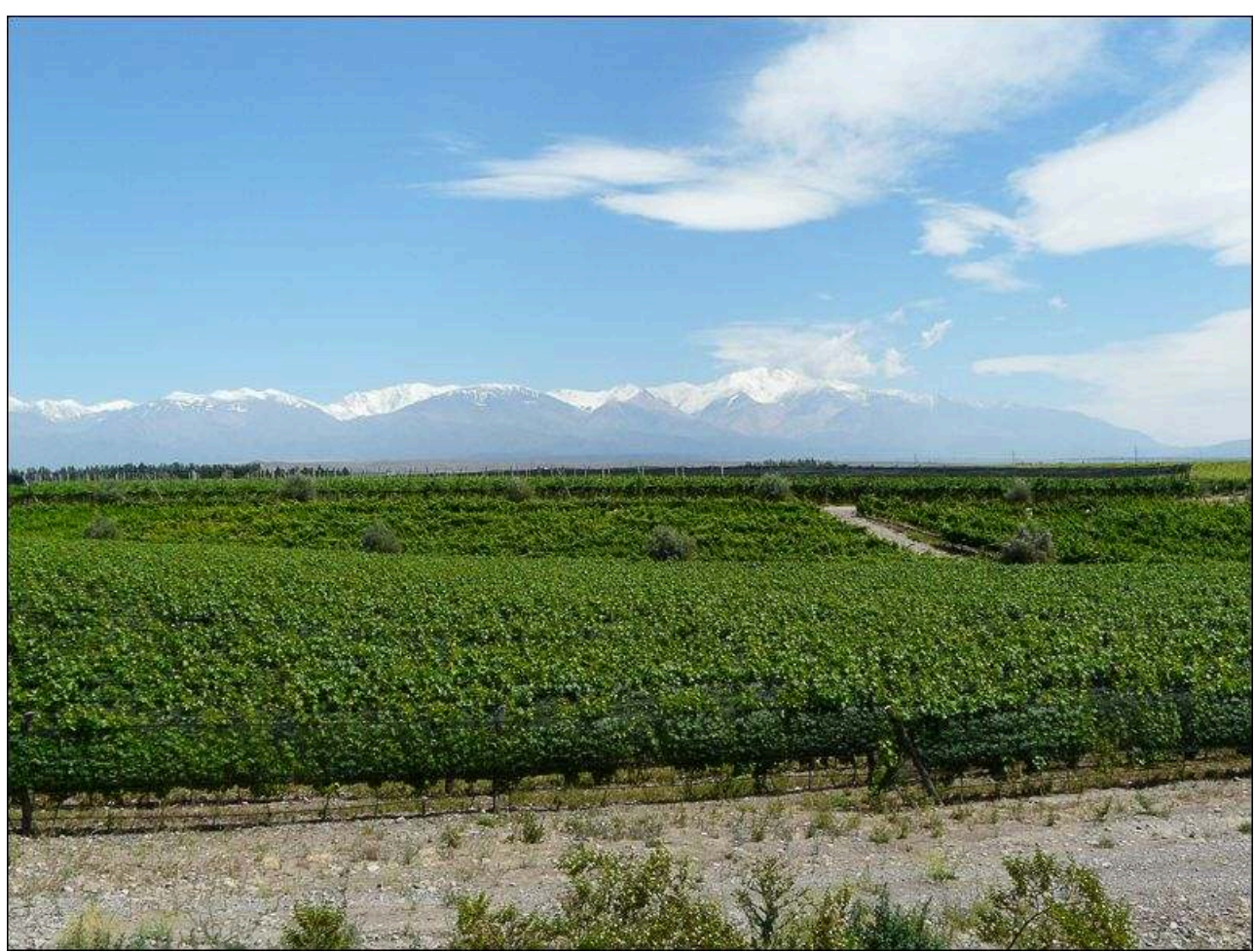

Source : Émilie Lavie, novembre 2014

Illustration 9 - Irrigation d'oliviers en goutte-à goutte, Gabès, Tunisie

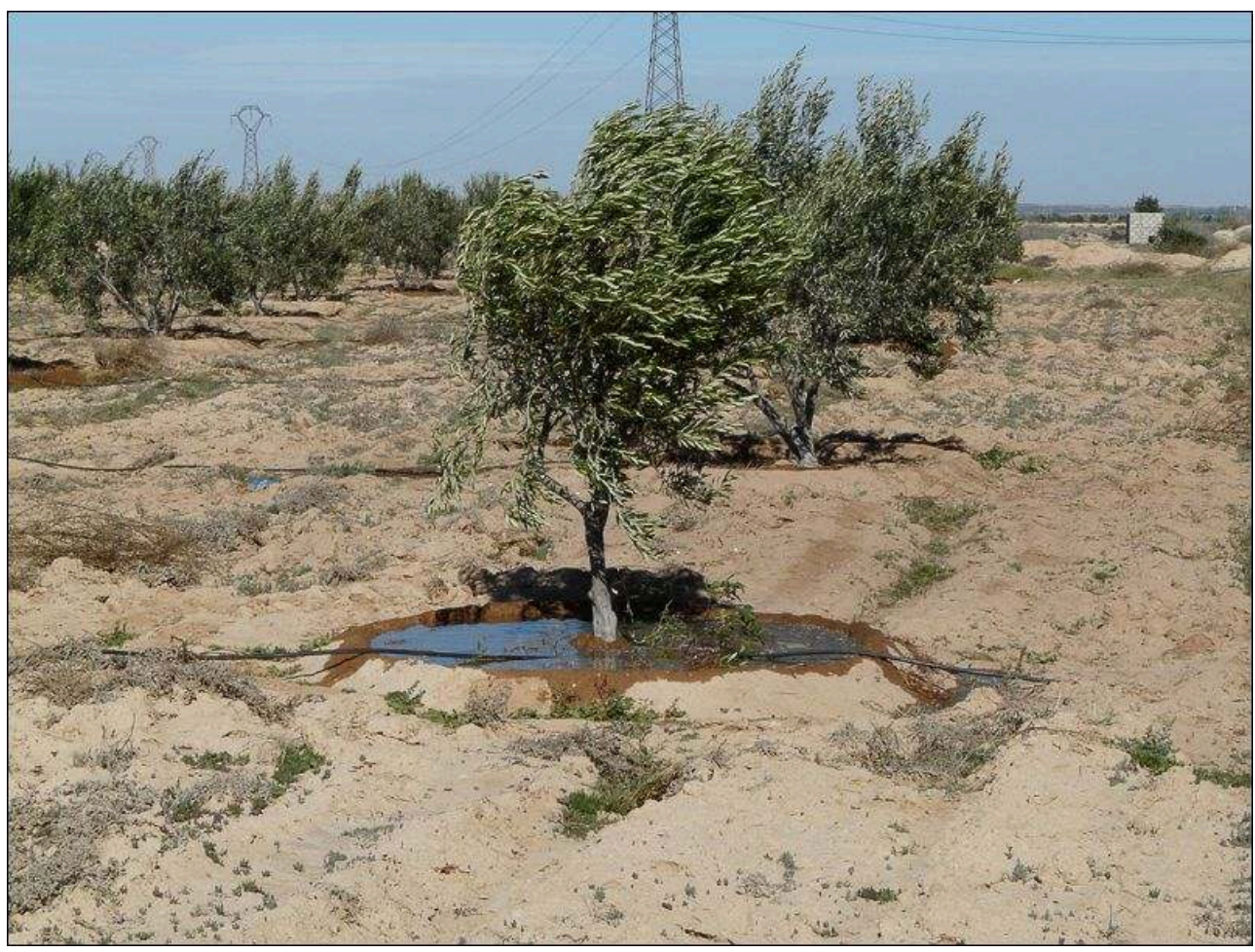

Source : Émilie Lavie, novembre 2017. 


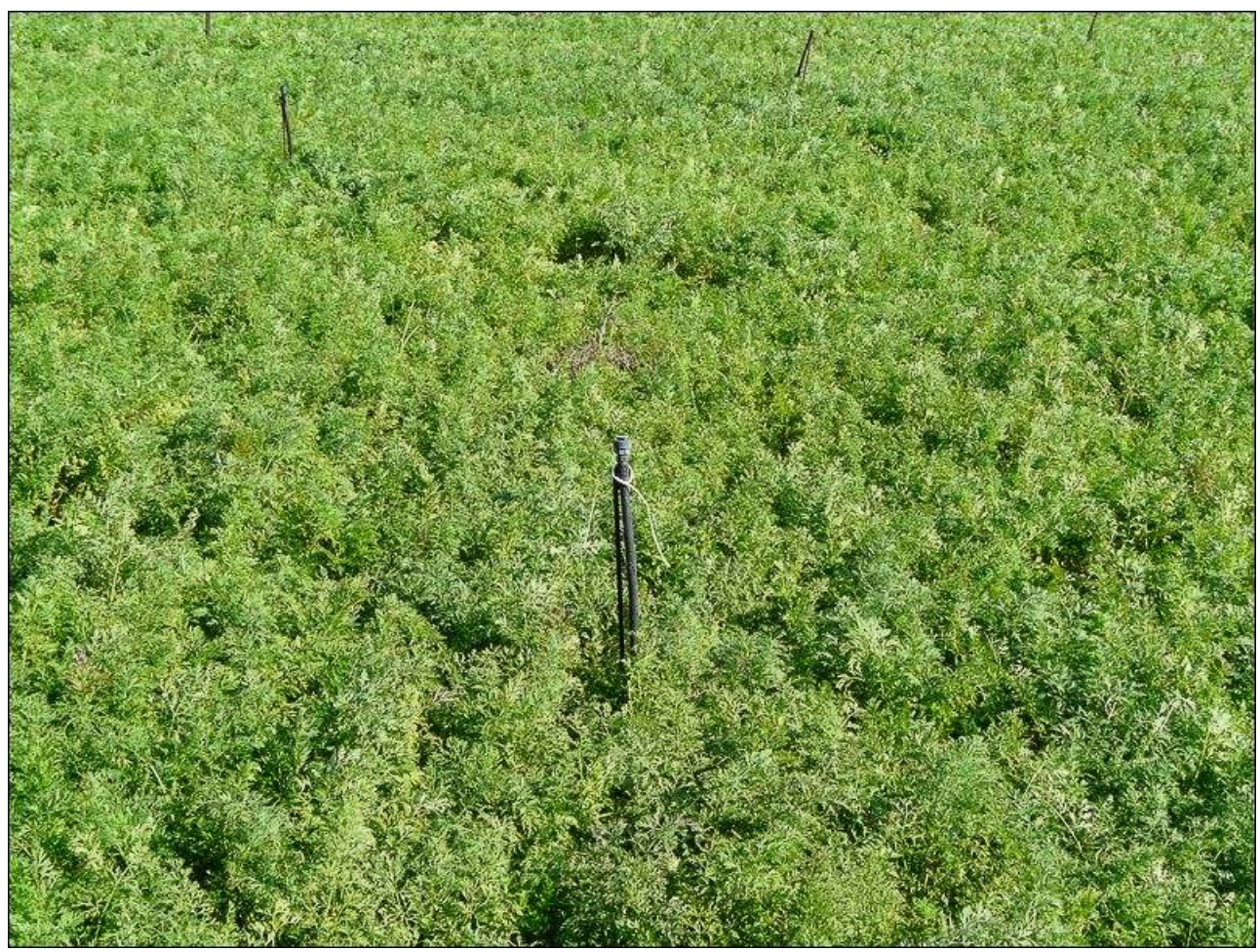

Source : Émilie Lavie, novembre 2017.

\section{Des systèmes rendus invisibles}

L'irrigation pressurisée est donc relativement visible, si tant est que l'on s'approche quelque peu des parcelles. Dans les oasis, seule la présence d'eau peut expliquer la végétation, toute culture est évidemment irriguée ; charge donc au/à la chercheur.e de trouver d'où vient l'eau et comment elle est apportée. Ce travail est réalisable en pratiquant des enquêtes si les gestionnaires sont enclins à donner des informations, ou par des relevés de de terrain, si tant est que la superficie de la zone à cartographier ne soit pas trop grande, auquel cas la photo-interprétation ou la télédétection sont de bons outils. Une difficulté survient lorsque l'on travaille dans des domaines qui ne sont pas arides. Les cultures peuvent être pluviales, à savoir non-irriguées, mais peuvent tout aussi bien l'être. Comment alors connaître le caractère irrigué ou non d'une parcelle ou d'un secteur?

Un premier biais est celui de la pluralité des fonctions de certains canaux. Par exemple, le Canal du Midi en France n'a pas été construit pour l'irrigation, mais il participe à celle de la zone dite de la Ganguise dans le Lauragais, et du Minervois audois, fonction qui n'est pas du tout évidente lorsqu'on parcourt ses rives puisque la plupart des prises sont situées sous l'eau. Une autre raison est l'enterrement des grands canaux. La Société d'Aménagement Régional BRL, sous concession de la Région Occitanie, met en place l'extension du canal P. Lamour qui dérive les eaux du Rhône vers le Languedoc et le Roussillon. Le canal actuel est visible jusqu'à Montpellier, je l'ai surligné en violet sur l'illustration 11. Mais son extension dans le cadre du projet Aqua Domitia est réalisée de manière souterraine. Les images satellitaires prises en 2014 (voir illustration 11) 
montrent les parcelles travaillées à gauche, on peut alors suivre l'extension du réseau général. À une échelle plus fine, BRL met à disposition la cartographie de ses réseaux principaux (cartographie du hautlllustration), mais les tuyaux sont souterrains et aujourd'hui invisibles sur le terrain (image de 2017). Seules quelques pompes en bord de route permettent de différencier des parcelles irriguées.

Illustration 11 - Irrigation dans l'Hérault et travaux d'extension du canal P. Lamour

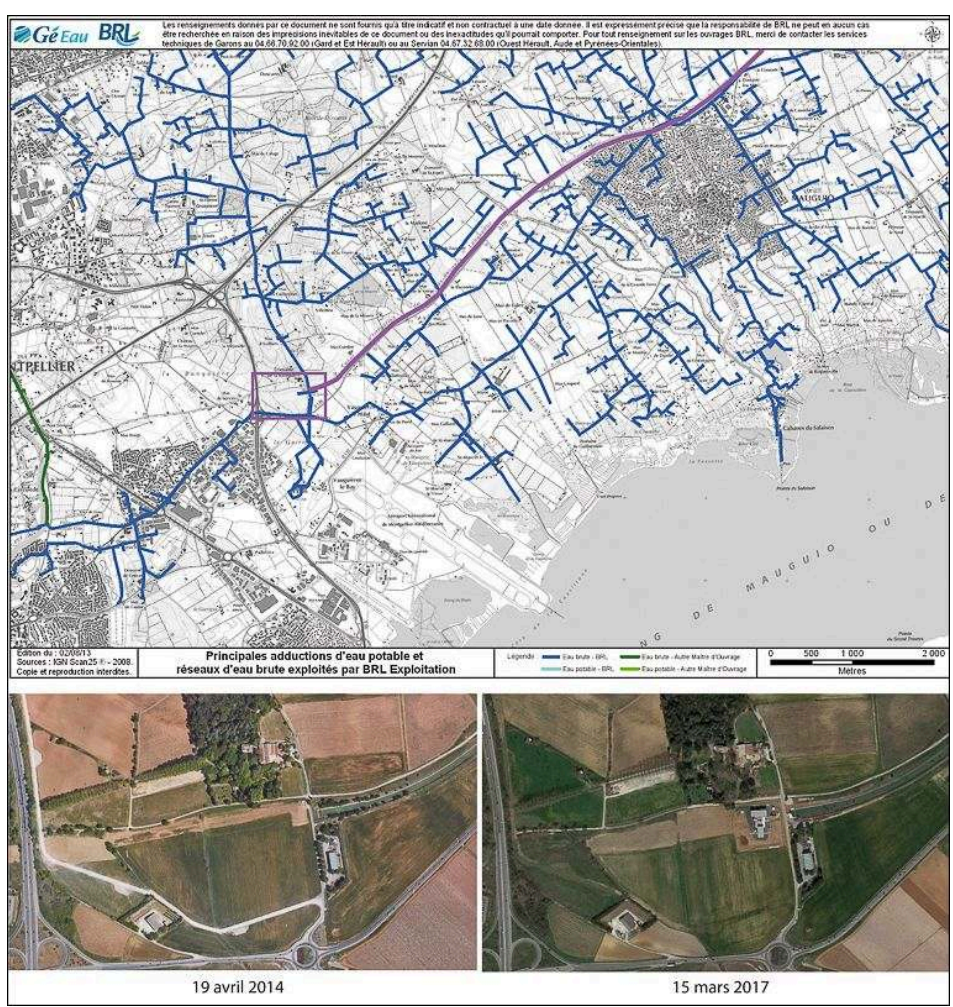

Sources : cartographie groupe BRL (modifiée) ; images Google Earth.

Enfin, parce qu'il est encore plus économe en eau (Mailhol et al., 2013), parce qu'il est moins contraignant en entretien, qu'il ne se casse pas sous l'effet de la grêle ou du passage des machines, le goutte-à-goutte enterré commence à se développer. Lors d'un terrain dans le Minervois, j'ai ainsi appris que certains secteurs étaient irrigués alors que les tuyaux sont invisibles. Sur l'illustration 12, la photo de droite montre des vignes à distance d'une source d'eau, sans tuyau apparent, ni au pied des ceps ni en bout de parcelle comme c'est parfois le cas. Les pompes sont très peu visibles, comme celle sur la photo de gauche à $500 \mathrm{~m}$ des routes. Dans ce cas d'un petit groupement d'agriculteurs, aucune cartographie n'existe en libre accès. Finalement, ni les relevés de terrain ni l'analyse d'images ne sont utilisables ici, seules les enquêtes auprès des irrigants voire des gestionnaires, nous ont permis de découvrir la réalité de l'extension de l'irrigation dans ce secteur (Lavie et al., 2018). 
Illustration 12 - Irrigation peu visible dans le bassin de la Valsèque, Minervois, France

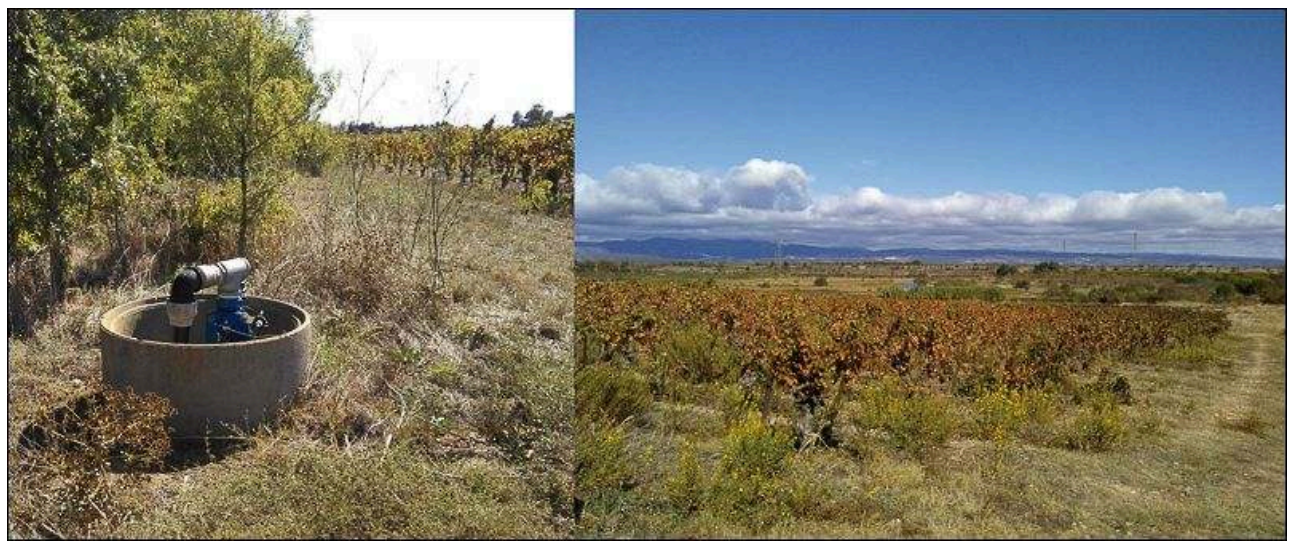

Source : Émilie Lavie, octobre 2017.

\section{Conclusion}

Cette analyse des chemins de l'eau d'irrigation à travers une série de photos et de cartes, dans des milieux divers, depuis les sources jusqu'aux parcelles, nous permet de démontrer la pluralité des systèmes d'irrigation, et surtout les trajectoires qu'ils ont connues. Très visibles, les infrastructures d'irrigation tendent à le devenir de moins en moins, tout comme d'autres usages comme l'hydroélectricité, l'industrie, et surtout l'eau potable et l'assainissement. Les méthodes de cartographie des réseaux, utiles pour comprendre les enjeux, doivent se renouveler. Elles ne passeront plus systématiquement par l'usage des images satellitaires ou des observations de terrain ; la disponibilité de cartographies lorsque les réseaux sont publics ou en délégation de service public est une première source de données, les enquêtes de terrain auprès des agriculteurs permettent de compléter ses manques. Finalement c'est en étudiant les jeux de pouvoir concernant la distribution de l'eau d'irrigation dans le Minervois que j'ai pu réaliser la cartographie, et non par la cartographie du chemin de l'eau que j'ai pu déterminer les jeux de pouvoir.

\section{BIBLIOGRAPHIE}

Aspe C., 2012. Introduction. In Aspe C. (coord.), De l'eau agricole à l'eau environnementale. Résistance et adaptation aux nouveaux enjeux de partage de l'eau en Méditerranée. Paris, Quae, p. 7-17.

Bruckmann L., 2016. L'intégration des zones inondables dans la gestion de l'eau et le développement de l'irrigation d'une vallée fluviale sahélienne. Le cas des terres de décrue de la moyenne vallée du Sénégal. Thèse de l'Université Paris Diderot (Paris 7) Sorbonne Paris Cité, [en ligne] https://hal.archives-ouvertes.fr/tel-01494483/ 
Cantor L.M., 1968. A world geography of irrigation. Edimbourg/Londres, Editions Olivier \& Boyd, p. 252.

Lavie E., Morábito J-A., Salatino S-E., 2017. The transformation of the Oases of Mendoza (Argentina): How the provincial socio-spatial structure was reversed by the crises of the 1980s and 2000s. In Lavie E., Marshall A. (Dir.) Oases and globalisation: ruptures and continuities. Springer International XV, p. 227-242.

Lavie E., Guillemot M., Mangeret C., 2018. Le futur espace hydraulique minervois. Méditerranée, Varia [en ligne].

Mainguet M., 2003. Les pays secs : environnement et développement. Paris, Ellipses, p. 152.

Marshall A., 2011. Terres gagnées et terres perdues : conséquences environnementales de l'essor de l'agro-industrie dans un désert de piémont. Le cas de l'oasis de Virú, Pérou. Bulletin de l'Institut français d'études andines, Vol. 40, n² 2, p. 375-396.

Marshall A., Lavie E., 2017. Introduction. In Lavie E., Marshall A. (dir.), Oases and globalisation: ruptures and continuities. Springer International XV. Hors numérotation.

Marshall A., 2014. Apropiarse del desierto, agricultura globalizada y dinámicas socioambientales en la costa peruana, el caso de los oasis de Virú e Ica-Villacuri. Lima, Editions de l'IFA-IRD, 417 p.

Mailhol J., Ruelle P., Dejean C., Rosique P., 2013. Le goutte-à-goutte enterré : une solution innovante pour irriguer sous conditions restrictives en eau. Sciences Eaux \& Territoires, Vol. 11, $\mathrm{n}^{\circ}$ 2, p. 26-29 [En ligne]. https://www.cairn.info/revue-sciences-eaux-et-territoires-2013-2page-26.htm

\section{AUTEUR}

\section{ÉMILIE LAVIE}

Ėmilie Lavie, emilie.lavie@univ-paris-diderot.fr, est maître de conférences à l'Université ParisDiderot, Sorbonne-Paris-Cité, et membre de l'UMR PRODIG. Elle a publié récemment :

- Lavie Ė., Marshall A. (dir.), (2017). Oases and globalization: ruptures and continuities. New York, Springer International, $255 \mathrm{p}$

- Lavie Ė. (2016). Le sabīl : marquer l'Islam dans la ville par des points d'eau. GéoConfluences [En ligne]. http://geoconfluences.ens-lyon.fr/informations-scientifiques/dossiers-thematiques/faitreligieux-et-construction-de-l-espace/le-sabil

- Redon M., Magrin G., Chauvin E., Perrier-Bruslé L., Lavie Ė. (dir.), 2015. Ressources mondialisées. Essai de géographie politique. Paris, Publications de la Sorbonne, $336 \mathrm{p}$. 